\title{
Preoperative contrast-enhanced computed tomographic characterisation of pancreatic cystic lesions: A prospective study
}

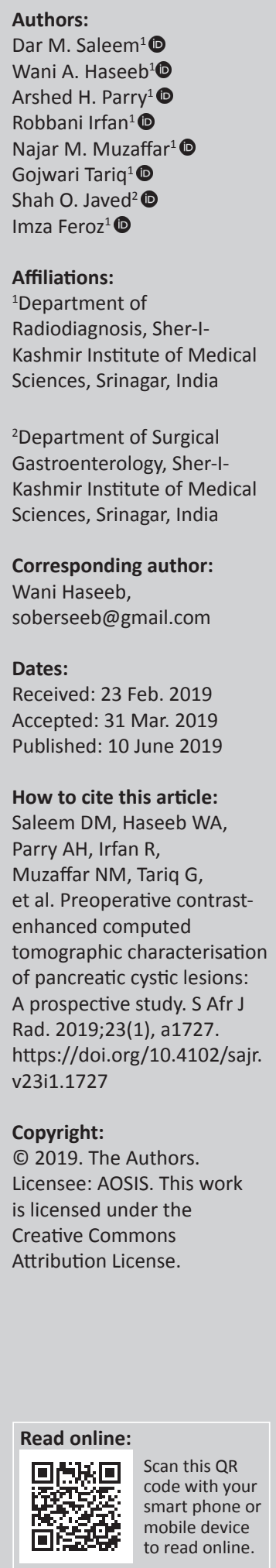

Authors:

Dar M. Saleem 1

Robbani Irfan'

Shah O. Javed ${ }^{2}$ (I)

Imza Feroz ${ }^{1}$

Kashmir Institute of Medica

${ }^{2}$ Department of Surgical

Gastroenterology, Sher-I-

Kashmir Institute of Medica

Corresponding author:

Wani Haseeb,

Dates:

Received: 23 Feb. 2019

Accepted: 31 Mar. 2019

How to cite this article:

Saleem DM, Haseeb WA

Parry AH, Irfan R,

et al. Preoperative contrast-

enhanced computed

tomographic characterisation

A prospective study SAfr I

Rad. 2019;23(1), a1727.

https://doi.org/10.4102/sajr

v23i1.1727

Copyright:

(n)
Background: Characterisation of pancreatic cystic lesions has a direct role in their management and computed tomography is the mainstay of investigation for diagnosing and characterising them.

Objectives: The aim of this study was to prospectively assess the diagnostic accuracy of contrast-enhanced computed tomography (CECT) in preoperative characterisation of pancreatic cystic lesions with histopathology as the reference standard.

Method: A total of 38 patients with cystic pancreatic lesions diagnosed after clinical, laboratory and sonographic evaluation, irrespective of age, were preoperatively evaluated with CECT. Images were reviewed for the general characteristics of the lesions on precontrast and portal venous phase images and overall diagnostic accuracy calculated. Imaging findings were compared with histopathology, or cytology and/or intra-operative findings.

Results: Serous cystadenoma (SCA) was the most common cystic pancreatic lesion found in $31.6 \%$ of patients followed by mucinous cystadenoma (MCA) (26.3\%), solid pseudo-papillary tumour (SPT) (21.1\%) and intra-ductal papillary mucinous neoplasm (IPMN) (10.5\%). Three patients $(7.9 \%)$ had simple cysts and one patient $(2.6 \%)$ had a lymphangioma. The diagnostic accuracy of CECT for pancreatic cystic lesions was found to be $72.5 \%$

Conclusion: The diagnostic accuracy of computed tomography (CT) was high for SCA, IPMN and pancreatic cysts, and low for MCA and SPT. Combination of a multiloculated cystic lesion with locule size of less than $20 \mathrm{~mm}$, septal enhancement with relative lack of wall enhancement, central scar and lobulated outline are highly specific for SCA. Unilocular or macro-cystic pattern with locule size of more than $20 \mathrm{~mm}$, female gender and wall enhancement with smooth external contour are pointers towards MCA. Solid cystic pancreatic head lesions in young females may be suggestive of SPT. A dilated main pancreatic duct in a cystic lesion with internal septations may point towards IPMN. Fluid attenuation lesions with imperceptible non-enhancing wall indicate pancreatic cysts. Lastly, pseudocysts and neuroendocrine tumours with cystic components are great mimickers of pancreatic cystic lesions, and a history of pancreatitis and hormonal profile of patients should always be sought.

Keywords: Serous cystadenoma; mucinous cystadenoma; solid pseudo-papillary tumour; intra-ductal papillary mucinous neoplasm; simple pancreatic cyst; pancreatic lymphangioma.

\section{Introduction}

Pancreatic lesions can be either solid, cystic or solid-cystic in nature, either of which can be benign, borderline or malignant. Most cystic lesions of the pancreas are benign. ${ }^{1,2,3}$ Characterisation becomes the sine qua non in distinguishing pancreatic cystic lesions as it has a direct bearing on their management, for example, to differentiate true cystic neoplasms from pancreatic pseudocysts. Serous cystadenomas (SCAs), mucinous cystic neoplasms and intra-ductal papillary mucinous neoplasms constitute more than $90 \%$ of primary cystic pancreatic neoplasms. ${ }^{2}$ Simple cysts and SCAs are benign and, if asymptomatic, can be safely followed. On the contrary, mucinous neoplasms are potentially malignant, justifying their surgical resection. ${ }^{1,2,3}$ Cystic pancreatic lesions are usually found incidentally on imaging studies performed for other reasons, and as many as 35\% of patients are totally asymptomatic at the time of diagnosis. ${ }^{1,2,3}$ 
Similar to Bosniak's classification for renal cysts, a radiological classification based on imaging features of pancreatic cystic lesions has been suggested. ${ }^{4}$ Pancreatic cystic lesions include unilocular cysts, micro-cystic lesions, macro-cystic lesions and mixed cystic lesions with a solid component. Fine needle aspiration provides a tissue diagnosis, but is often nondiagnostic because of sampling error ${ }^{5}$ and various limitations. ${ }^{6}$ Its sensitivity for diagnosis of cystic lesions is much lower than for solid lesions. ${ }^{7,8}$ Imaging modalities such as ultrasonography (US), computed tomography (CT), magnetic resonance imaging (MRI), positron emission tomography and endoscopic US play a key role in characterisation, staging, surgical planning and assessment for treatment. ${ }^{9,10,11}$ The best approach to obtain an exact preoperative diagnosis is the combined evaluation of all available clinical, serological, radiological and biopsy findings.

Ultrasonography is not an ideal screening tool for the detection of pancreatic masses because of its relatively low sensitivity. ${ }^{12,13}$ The major limitation of endoscopic US is its inability to stage disease beyond the pancreas, thus it is generally used as an adjunct to or after multidetector computed tomography (MDCT). Magnetic resonance imaging is useful for iso-enhancing pancreatic masses that are not directly seen on CT. ${ }^{14}$ Susceptibility of MRI to significant image degradation by respiratory motion artefact is even more so in contrast studies, which is often critical for characterising pancreatic lesions, limits its diagnostic capability. ${ }^{15,16}$ In spite of advances in MRI in abdominal imaging, CT is still the preferred imaging modality for both initial detection and characterisation of cystic pancreatic lesions, especially the macro-cystic ones. ${ }^{17}$ Computed tomography is an excellent imaging modality for pancreatic cystic lesions because of its widespread availability and ability to detect cysts. ${ }^{18}$

This study was undertaken to evaluate the possible role of $\mathrm{CT}$ in the preoperative characterisation of pancreatic lesions, on the basis of various morphological characteristics, because preoperative fine needle aspiration cytology may lack the desired diagnostic accuracy, more so in cases of cystic lesions.

\section{Materials and methods}

Prior to imaging, the risks and benefits were discussed with each patient and informed consent taken. Patients with cystic pancreatic lesions diagnosed after clinical, laboratory and sonographic evaluation, irrespective of age, were included in this study. Patients with inflammatory, parasitic or purely solid pancreatic lesions based on imaging, clinical and laboratory profiles and patients with deranged renal function tests, contrast allergy and pregnancy were excluded from the study. To characterise the pancreatic cystic lesions, a real-time ultrasound examination of the abdomen was done, followed by contrast-enhanced CT (CECT).

Ultrasound was done using a $3.5 \mathrm{MHz}$ curvilinear and 7.5 $\mathrm{MHz}$ linear array probe on an Aloka Prosound SSD-3500SX machine. All CT examinations were performed on a Siemens
Somatom Sensation 64. Contrast-enhanced CT of the abdomen was performed on all patients. The anterior cubital vein of the patients was cannulated using an 18G intravenous cannula. A pressure injector was used to inject $120 \mathrm{~mL}-150$ $\mathrm{mL}$ of contrast (omnipaque) as per body weight. After obtaining the topogram and non-contrast images of the abdomen, a portal venous phase CT was performed 40-60 s after the start of contrast injection. On a workstation, multiplanar coronal, sagittal and axial reconstructions were performed. Analysis of CT images was performed on a picture archiving and communication system (PACS) workstation monitor by an experienced radiologist with more than 15 years of abdominal imaging experience. The radiologist was blinded to the histopathological and surgical findings, but not to the clinical history of the patients. Images were reviewed for the general characteristics of the lesions on pre-contrast and portal venous phase images. Imaging findings were compared with histopathology/cytology and/ or intra-operative findings. The final diagnosis was confirmed by histopathology/cytology in all cases.

\section{Statistical analysis}

The data were analysed using statistical software SPSS v20 and STATA v11. Categorical variables were described in terms of frequency and percentage and the continuous variables in terms of descriptive statistics like mean, standard deviation (SD), minimum, maximum and range. Also, the sensitivity, specificity and accuracy were calculated. All the results were determined at the $5 \%$ significance level.

\section{Ethical consideration}

This was a prospective observational study conducted between October 2015 and December 2018, with approval from the Institutional Ethical Committee (IEC) (No. SIMS 1 31/IEC-SKIMS/2015-75) and a final sample size of 38.

\section{Results}

Of the 38 patients diagnosed with cystic pancreatic lesions on imaging, 12 patients (31.6\%) had SCA, 10 patients $(26.3 \%)$ had mucinous cystadenomas (MCA), $8(21.1 \%)$ had solid pseudo-papillary tumours (SPT), 4 (10.5\%) patients had intra-ductal papillary mucinous neoplasms (IPMN), 3 patients $(7.9 \%)$ had simple cysts and 1 patient $(2.6 \%)$ had a lymphangioma. The age group and mean age (in years) at the time of diagnosis and gender distribution of various pancreatic cystic lesions are shown in Table 1. Distribution with respect to anatomic location in the pancreas is shown in Table 2. The mean size of a SCA on CT was $3.5 \mathrm{~cm}$ and that of MCA was $7.3 \mathrm{~cm}$; mean size of SPT was $5.3 \mathrm{~cm}$.

The enhancement pattern on CECT was defined as either absent or enhancement of the wall, septae, both septae and wall and solid-cystic with enhancement of solid component, as shown in Table 3. Excluding SPT that has a solid component, Table 4 shows the loculation pattern and the size of the locules (in multilocular cystic lesions) in the truly cystic pancreatic lesions. Intra-hepatic biliary radicles and 
TABLE 1: Age, mean age (in years) and gender distribution of various pancreatic cystic lesions at the time of diagnosis.

\begin{tabular}{|c|c|c|c|c|c|c|c|c|c|}
\hline \multirow[t]{3}{*}{ Final diagnosis } & \multicolumn{6}{|c|}{ Age (years) } & \multirow{3}{*}{$\begin{array}{l}\text { Mean age } \\
\text { (years) }\end{array}$} & \multicolumn{2}{|c|}{ Gender } \\
\hline & \multicolumn{2}{|c|}{$<30$} & \multicolumn{2}{|c|}{$30-50$} & \multicolumn{2}{|c|}{$>50$} & & Male & Female \\
\hline & $n$ & $\%$ & $n$ & $\%$ & $n$ & $\%$ & & & \\
\hline SCA & 4 & 33.3 & 6 & 50 & 2 & 16.7 & 44.5 & 2 & 10 \\
\hline MCA & 2 & 20 & 4 & 60 & 2 & 20 & 47.0 & 0 & 10 \\
\hline SPT & 6 & 75 & 2 & 25 & 0 & 0 & 26.5 & 0 & 8 \\
\hline IPMN & 0 & 0 & 2 & 50 & 2 & 50 & 56.0 & 4 & 0 \\
\hline Simple cyst & 3 & 100 & 0 & 0 & 0 & 0 & 27.0 & 1 & 2 \\
\hline Lymphangioma & 0 & 0 & 0 & 0 & 1 & 100 & 55.0 & 0 & 1 \\
\hline
\end{tabular}

SCA, serous cystadenoma; MCA, mucinous cystadenoma; SPT, solid pseudo-papillary tumour; IPMN, intra-ductal papillary mucinous neoplasm.

TABLE 2: Distribution of pancreatic cystic lesions as per the anatomic site.

\begin{tabular}{|c|c|c|c|c|c|c|}
\hline \multirow[t]{3}{*}{ Diagnosis } & \multicolumn{6}{|c|}{ Site of the lesion in pancreas } \\
\hline & \multicolumn{2}{|c|}{ Head or uncinate } & \multicolumn{2}{|c|}{ Body } & \multicolumn{2}{|c|}{ Tail } \\
\hline & $n$ & $\%$ & $n$ & $\%$ & $n$ & $\%$ \\
\hline SCA & 8 & 66.7 & 4 & 33.3 & 0 & 0 \\
\hline MCA & 0 & 0 & 2 & 20 & 8 & 80 \\
\hline SPT & 6 & 75 & 0 & 0 & 2 & 25 \\
\hline IPMN & 2 & 50 & 2 & 50 & 0 & 0 \\
\hline Simple cyst & 0 & 0 & 3 & 100 & 0 & 0 \\
\hline Lymphangioma & 0 & 0 & 1 & 100 & 0 & 0 \\
\hline
\end{tabular}

SCA, serous cystadenoma; MCA, mucinous cystadenoma; SPT, solid pseudo-papillary tumour; IPMN, intra-ductal papillary mucinous neoplasm.

TABLE 3: Enhancement pattern on computed tomography of various pancreatic cystic lesions.

\begin{tabular}{lccccc}
\hline Final diagnosis & \multicolumn{5}{c}{ Enhancement } \\
\cline { 2 - 6 } & Nil & Wall & Septal & Wall and septal & Solid cystic \\
\hline SCA & 2 & 0 & 10 & 0 & 0 \\
MCA & 0 & 6 & 0 & 4 & 0 \\
SPT & 0 & 0 & 0 & 0 & 8 \\
IPMN & 0 & 0 & 4 & 0 & 0 \\
Simple cyst & 3 & 0 & 0 & 0 & 0 \\
Lymphangioma & 0 & 0 & 1 & 0 & 0 \\
\hline
\end{tabular}

SCA, serous cystadenoma; MCA, mucinous cystadenoma; SPT, solid pseudo-papillary tumour; IPMN, intra-ductal papillary mucinous neoplasm.

TABLE 4: Loculation pattern and size of pancreatic cystic lesions.

\begin{tabular}{|c|c|c|c|c|c|c|}
\hline \multirow[t]{2}{*}{ Lesion } & \multicolumn{2}{|c|}{ Unilocular } & \multicolumn{2}{|c|}{$>1$ Locule } & \multicolumn{2}{|c|}{ Size of locules } \\
\hline & $n$ & $\%$ & $n$ & $\%$ & $\leq 20 \mathrm{~mm}$ & $>20 \mathrm{~mm}$ \\
\hline SCA & 2 & 16.7 & 10 & 83.3 & 10 & 0 \\
\hline MCA & 6 & 60 & 4 & 40 & 0 & 4 \\
\hline IPMN & 0 & 0 & 4 & 100 & 4 & 0 \\
\hline Simple cyst & 3 & 100 & 0 & 0 & 0 & 0 \\
\hline Lymphangioma & 0 & 0 & 1 & 100 & 1 & 0 \\
\hline
\end{tabular}

SCA, serous cystadenoma; MCA, mucinous cystadenoma; SPT, solid pseudo-papillary tumour; IPMN, intra-ductal papillary mucinous neoplasm.

common bile duct (CBD) were not dilated in any of the cystic lesions. Main pancreatic duct (MPD) dilatation was seen in the four patients $(100 \%)$ with IPMN and in two patients (20\%) with MCA; normal MPD seen in the rest of the cystic lesions.

Ten of the 12 cystic lesions diagnosed as SCA on CT proved to be SCA on histopathology examination (HPE) as well; one was diagnosed as a pseudocyst of pancreas and the other as MCA on HPE. Six of the 10 patients were correctly diagnosed as MCA on CT; three were found to be pseudocysts and one was a SCA on HPE or surgery. Five of the eight patients were correctly diagnosed as SPT on CT, while the other three proved to be neuroendocrine tumours with cystic components on HPE. All of the four cases of IPMN, three cases of simple cysts and one case of lymphangioma were correctly identified on CT. The diagnostic accuracy of CECT for pancreatic cystic lesions was found to be $72.5 \%$, with $57.16 \%$ and $83.89 \%$ as the lower and upper $95 \%$ confidence interval limits.

\section{Discussion}

Serous cystadenoma was the most common cystic pancreatic lesion found in $31.6 \%$ of patients in our study followed by MCA (26.3\%), SPT (21.1\%) and IPMN (10.5\%). Three patients (7.9\%) had simple cysts and one patient $(2.6 \%)$ had a lymphangioma.

Serous cystadenoma was found predominantly in females in our study (male:female ratio of 1:5) with mean age of 44.5 years, similar to the studies of Atalay et al. ${ }^{19}$ and Parra-Herran et al. ${ }^{20}$ Serous cystadenomas were predominantly located in the head or uncinate process (66.7\%) (Figure 1) and the body of pancreas (33.3\%). None were located in the pancreatic tail. Megibow et al. ${ }^{21}$ reported that serous cystic neoplasm is most often found in the pancreatic head. Atalay et al. ${ }^{19}$ reported 23 cases of SCAs of pancreas, all of which were present in the head and body of the pancreas. Of the 12 SCAs, $2(16.7 \%)$ were unilocular and 10 (83.3\%) were multiloculated, consistent with the study of Atalay et al. ${ }^{19}$ All the multiloculated SCAs had the size of the largest locule, less than $20 \mathrm{~mm}$. Johnson et al..$^{22}$ reported that on ultrasound, SCAs usually have more than six loculi that are less than $2 \mathrm{~cm}$ in diameter. Bhatt and Vaishnav ${ }^{23}$ found that SCAs were approximately $5 \mathrm{~cm}-6 \mathrm{~cm}$ in size with small internal cysts $<2 \mathrm{~cm}$, with septations. However, Curry et al. ${ }^{24}$ reported that the largest cyst in each tumour was smaller than $2 \mathrm{~cm}$ in only $14(64 \%)$ out of the 22 patients. Four (33.3\%) SCAs had a lobulated outline that is characteristic of SCA, ${ }^{18,25}$ while others had a regular outline. Central scar, a characteristic of SCA, ${ }^{18,25}$ was noted only in two (16.7\%) of the SCAs (Figure 1b). Torresan et al. ${ }^{26}$ reported that although seen in less than $20 \%$ of SCAs, demonstration of a central scar by CT or MRI is a highly diagnostic feature of a SCA. Calcification was seen in none of the 12 SCAs in our study. However, central calcification can be seen in SCAs within the fibrous stroma. ${ }^{25}$ Curry $^{24}$ reported central calcification in $10 \%$ of all cystic pancreatic lesions in their study (5/50), of which $80 \%$ were SCAs. The majority of SCAs in our study $(10 / 12)$ showed 

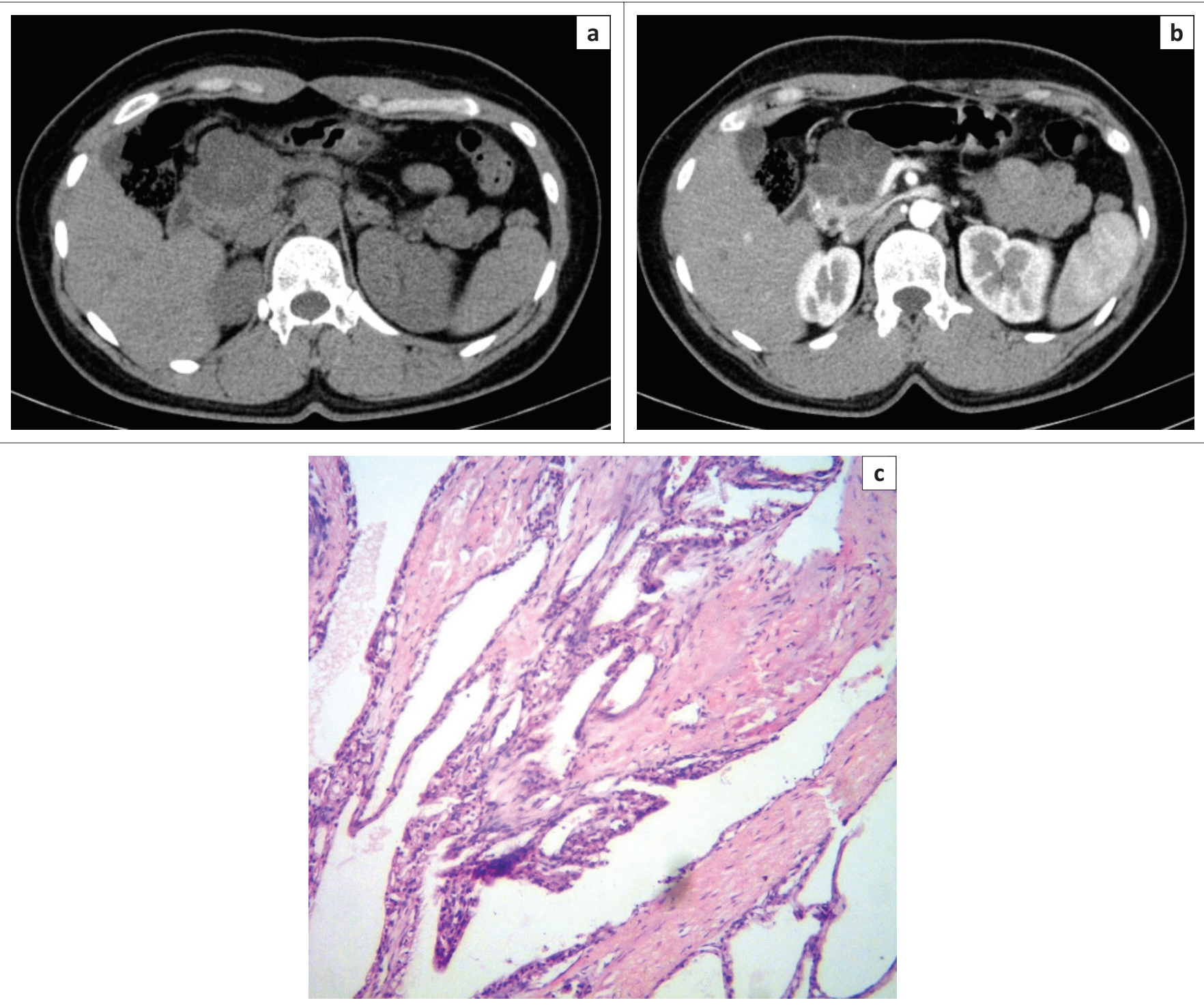

FIGURE 1: Axial non-contrast-enhanced computed tomography image (a) in a 37-year-old female patient showing fluid density lobulated lesion in the head of pancreas. Axial contrast-enhanced computed tomography image (b) in the same patient showing a multiloculated cystic mass in the head of pancreas with enhancing septations, a central stellate scar and no definite wall enhancement, suggestive of serous cystadenoma. High power magnification (40X) photomicrograph (c) of the same patient confirmed the diagnosis of micro-cystic serous cystadenoma.

septal enhancement (Figure 1b) on CECT, as also reported by Balthazar et al. ${ }^{27}$ Biliary radicles, CBD and MPD were not dilated in any SCAs in our study, which is consistent with the literature. Rarely, giant lesions can cause compression of the MPD or bile ducts. ${ }^{28}$ Histopathology examination revealed the two pancreatic cystic lesions presumptively diagnosed as SCAs on CT in our study to be pseudocyst of pancreas and MCA. Both these lesions were unilocular or oligocystic, and hence MCA and pseudocysts are close differentials.

Mucinous cystadenoma was the second most common pancreatic cystic lesion (26.3\%) with a mean age of 47 years at the time of diagnosis and seen exclusively in females in our study. They were predominantly located in tail (80\%) (Figure 2) and pancreatic body (20\%), with no lesions seen in head or uncinate process. Mean size of MCA $(7.3 \mathrm{~cm})$ was larger than SCA and SPT in our study. The findings are consistent with the majority of other studies ${ }^{19,20,29}$ The majority of MCAs were unilocular (60\%) with $40 \%$ appearing multilocular in our study. The size of the largest locule was $>20 \mathrm{~mm}$ in multiloculated lesions. Unilocular or macro-cystic pattern, ${ }^{30}$ with wall enhancement, is very helpful in diagnosing MCA on CT. Curry ${ }^{24}$ reported that the largest locule in each tumour was larger than $2 \mathrm{~cm}$ in 24 patients $(86 \%)$ with MCA. Mucinous cystadenomas showed a smooth external contour (Figure 2) in our study consistent with the world literature.$^{31}$ Calcifications were present in none of these lesions, although MCAs may have a peripheral eggshell calcification. ${ }^{25}$ Wall enhancement was seen in all cases of MCAs in our study on CECT (Figure 2b), with only $40 \%$ cases showing variable enhancement of septations. Cohen-Scali et al. $^{32}$ reported that the lack of wall enhancement was specific for macro-cystic SCA in comparison with MCA. Biliary radicles, $\mathrm{CBD}$ and $\mathrm{MPD}$ were not dilated in any MCA; however, Warshaw et al. ${ }^{31}$ reported that very rarely they can cause ductal obstruction, but do not communicate with the MPD. Three cases were misdiagnosed as MCA on CT, which turned out to be pseudocysts. Pseudocyst with wall 

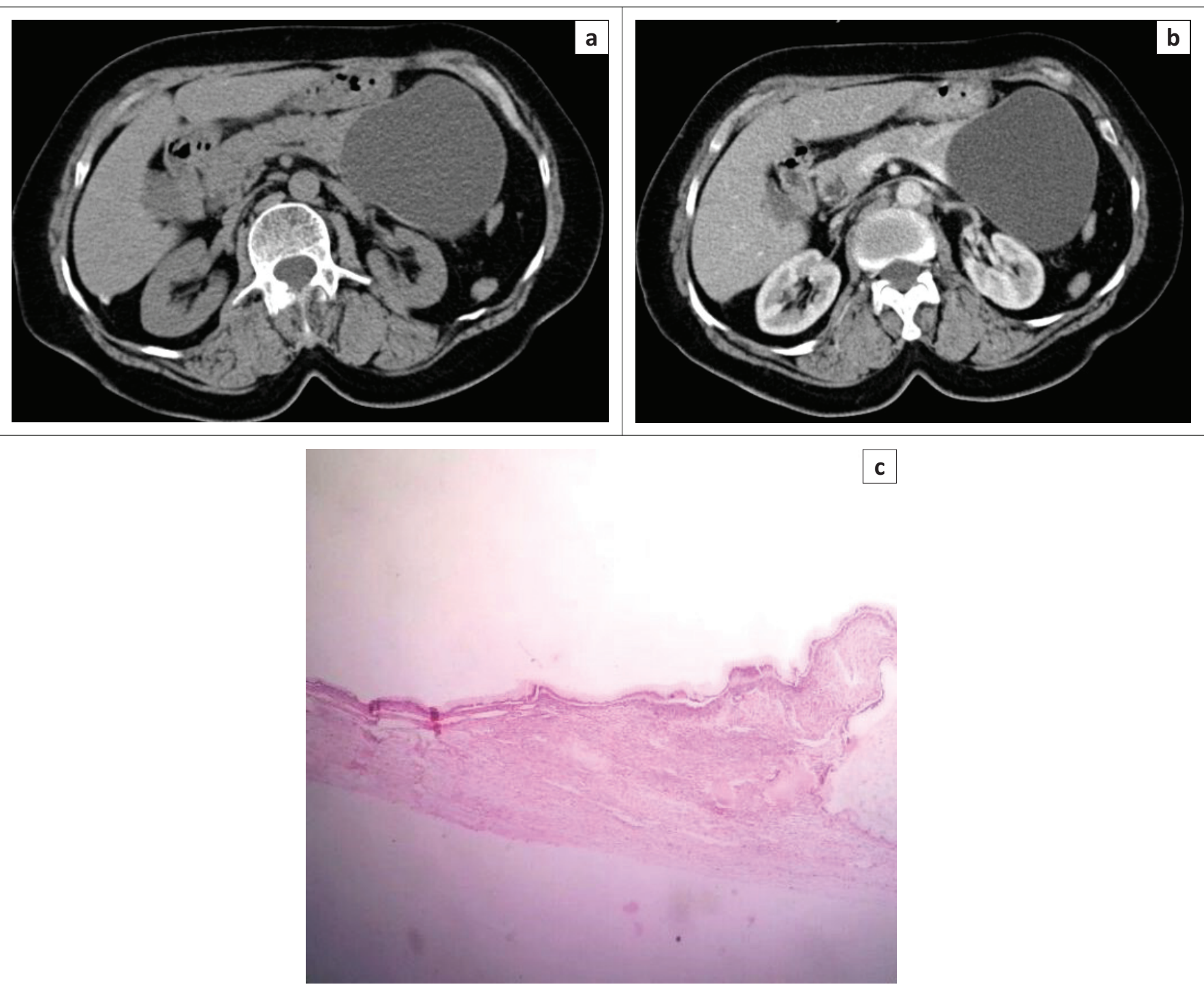

C

FIGURE 2: Axial non-contrast-enhanced computed tomography image (a) in a 49-year-old female patient showing large fluid attenuation cystic lesion in the tail of pancreas with a smooth external contour. Axial contrast-enhanced computed tomography image (b) in the same patient showing a non-enhancing cystic lesion in the tail of pancreas with subtle wall enhancement, suggestive of mucinous cystadenoma. Low power magnification (scanner 4X) photomicrograph (c) of the same patient confirmed the diagnosis of mucinous cystadenoma.

enhancement is a close differential for MCA on CT, especially if the past history of pancreatitis is not forthcoming. Because of significant wall enhancement, one unilocular SCA was wrongly labelled as MCA on CT.

Solid pseudo-papillary tumour constituted $21.1 \%$ of pancreatic lesions in our study with $75 \%$ of patients under the age of 30 years, with a mean age of 26.5 years and was seen exclusively in females. It was predominantly located in head of pancreas (75\%) (Figure 3) in our study, with only $25 \%$ cases in the tail region (Figure 4). These findings were in resonance with other studies. ${ }^{33-37}$ All the cases of SPT in our study had a solid cystic appearance on CT with an enhancing large solid component (Figures $3 b$ and $4 b$ ). None had a pure cystic appearance. Casedei et al. ${ }^{35}$ reported four cases of solid pseudo-papillary neoplasms and all four were solid welldefined masses. Alves et al. ${ }^{37}$ conducted a study on 10 pancreatic solid pseudo-papillary neoplasms, and found that on radiology the tumour was solid cystic or solid with almost equal frequency. This disparity of results in the SPT appearance in our study could be accounted for because of exclusion of purely solid lesions. Kehagias et al. ${ }^{38}$ reported that enhancing solid areas in SPT are typically peripheral in location, whereas cystic spaces are usually more centrally located. Calcification was an inconsistent finding with variable pattern seen in only 2 two (50\%) lesions in a peripheral location. Megibow et al. ${ }^{21}$ reported calcifications in $29 \%$ of SPTs. Common bile duct, MPD and the biliary tree were normal in all SPT cases consistent with the literature. ${ }^{39}$ No metastatic liver lesions were present in our study, although metastases can occur. ${ }^{40}$ Three of these lesions presumptively diagnosed as SPT on CT in our study turned out to be neuroendocrine tumours on HPE. We determined that non-functioning neuroendocrine tumours become large and undergo cystic change, thus closely resembling SPTs on cross-sectional imaging. Choi et $\mathrm{l}^{41}$ reported that SPTs with a minimal cystic component or no intra-tumoural haemorrhage are difficult to differentiate from islet cell tumours. 

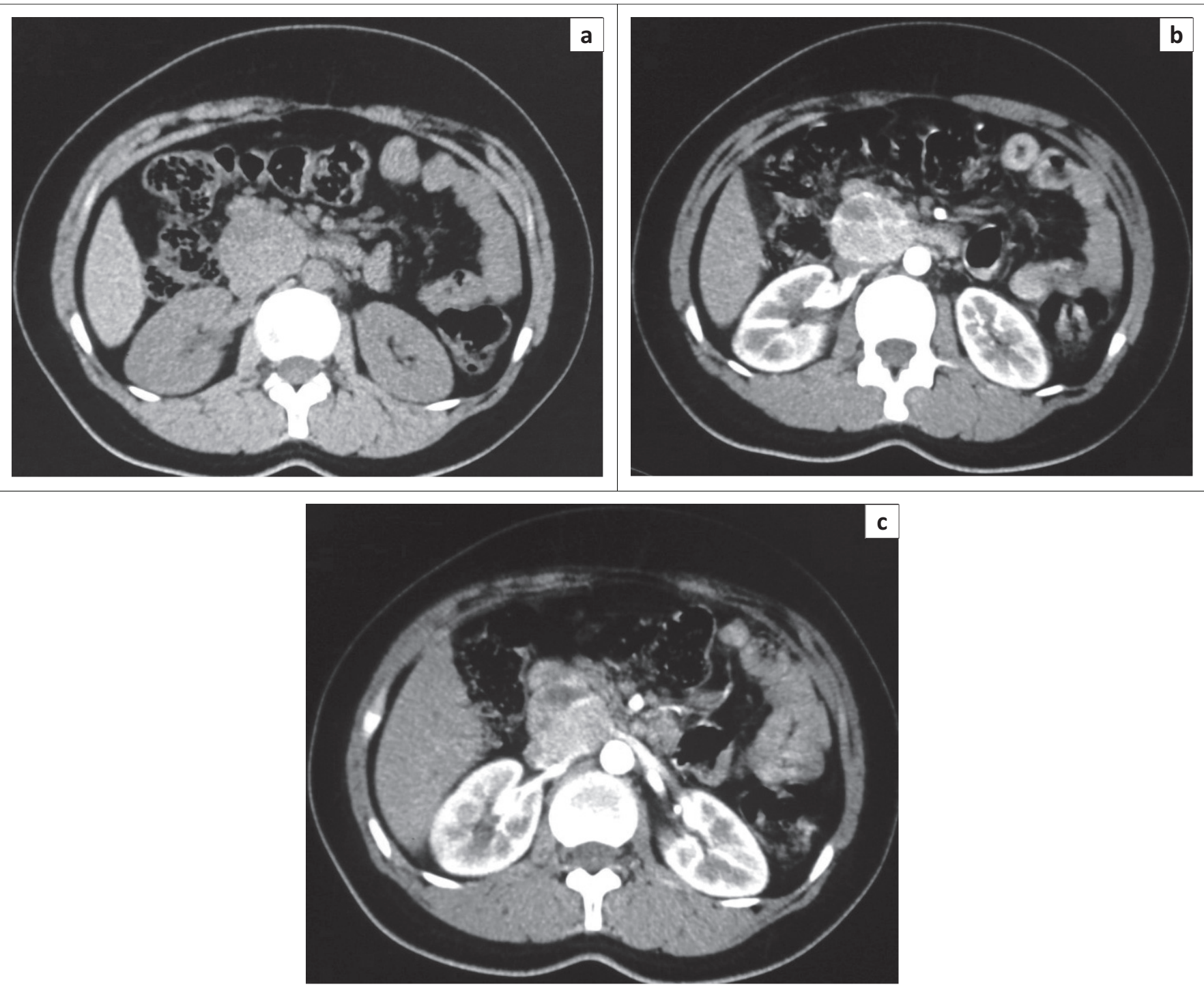

FIGURE 3: Axial non-contrast-enhanced computed tomography image (a) in a 26-year-old female patient showing a well-defined lesion in the pancreatic head or uncinate process with axial contrast-enhanced (b) and magnified post-contrast computed tomography image (c) showing an enhancing solid lesion with a non-enhancing component suggestive of solid pseudo-papillary tumour. Histopathology examination confirmed the same.

Intra-ductal papillary mucinous neoplasm was seen in $10.5 \%$ of our patients with a mean age of 56 years at the time of diagnosis and exclusively with a male distribution, consistent with the international literature reflecting a predominance in middle to elderly men. ${ }^{42}$ An equal number of lesions were seen in the head and the body region (Figure 5). Paal et $\mathrm{al}^{42}$ reported that 18 of 22 IPMNs were present in the head. The mean size of the lesions at sonography was $2.65 \mathrm{~cm}$, and at CT and pathology it was $2.5 \mathrm{~cm}$, which is consistent with the literature. ${ }^{43}$ All the IPMNs appeared as cysts with internal septa (Figure 5). The main pancreatic duct was dilated in all the four cases (Figure 5); however, communication of the cyst with the MPD was difficult to identify in three patients and was identified only in one patient with certainty. Procacci ${ }^{44}$ found that at US and $\mathrm{CT}$, branch duct tumours, which were mainly located at the uncinate process, were seen as fluid-filled masses with central septa and the pancreatic duct was dilated. Paal et al. ${ }^{42}$ conducted a study on 22 pancreatic IPMNs. Radiologically, the cases presented with inhomogeneous solid or cystic masses. However, a dilated pancreatic duct was present in all cases. We found that cystic pancreatic lesions with enhancing septa and dilated MPD in an elderly male are helpful pointers towards IPMN.

Simple pancreatic cysts constituted $7.9 \%$ of the pancreatic cystic lesions with fluid attenuation on CT, and no septations, solid component or calcifications were seen within them. The outline was smooth. No enhancement was seen on postcontrast CT (Figure 6). The main pancreatic duct and biliary tree were not dilated. These features are consistent with the world literature. ${ }^{45,46}$ The least common cystic pancreatic lesion was pancreatic lymphangioma, with only one case seen in our study. The 55-year-old woman showed a multicystic peripancreatic lesion closely abutting the pancreas and insinuating in between the surrounding structures with no mass effect. Few tiny calcific foci were also seen; however, no definite fat attenuation was noted. A presumptive diagnosis of pancreatic lymphangioma was made on CT and subsequently proved on HPE. 

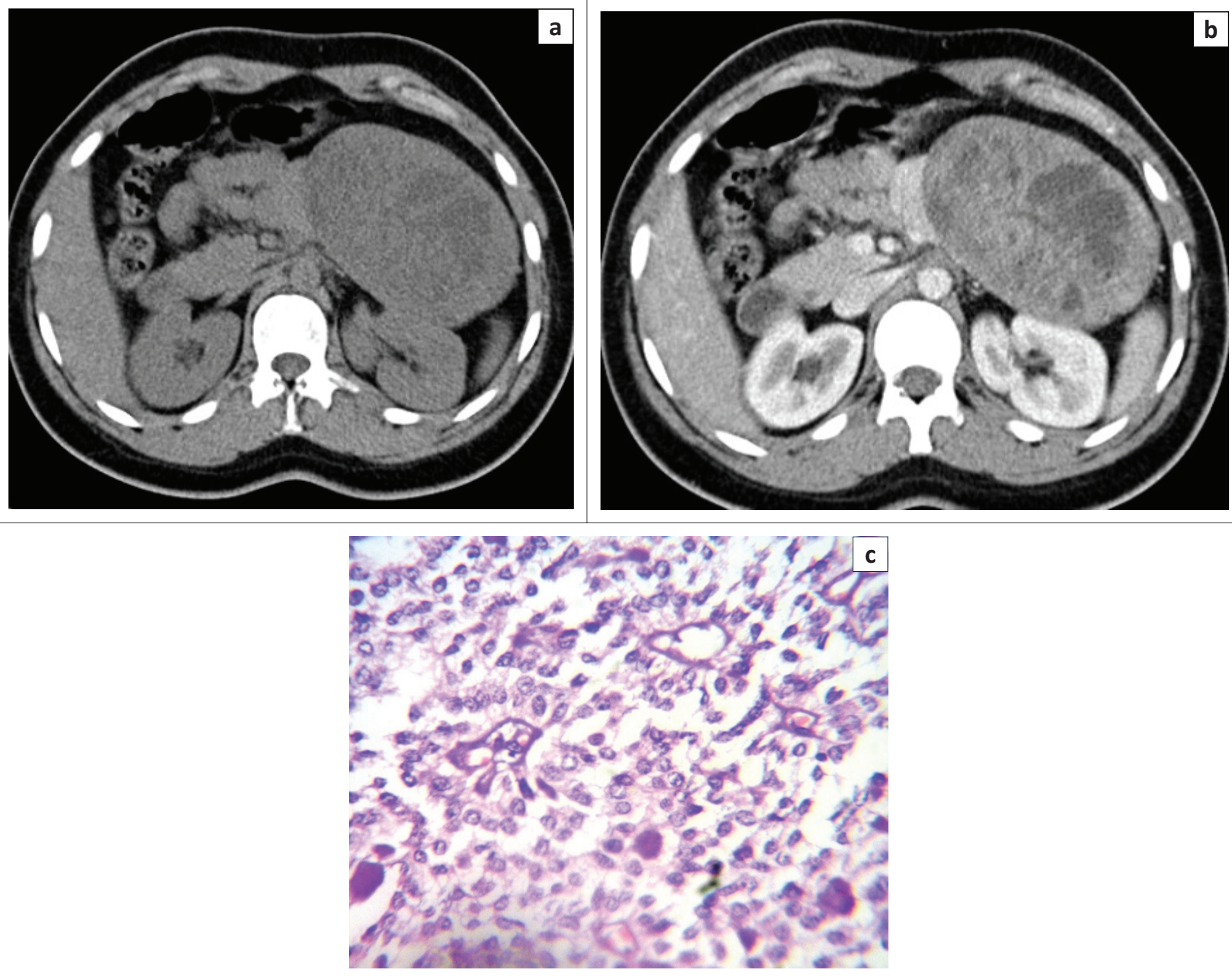

FIGURE 4: Axial non-contrast-enhanced computed tomography image (a) in a 31-year-old female patient showing a large lesion in relation to the tail of pancreas with a central hypodense area. Axial contrast-enhanced computed tomography image (b) in the same patient showing enhancement of the solid component of the lesion with non-enhancing areas suggestive of a solid pseudo-papillary tumour. High power magnification (40X) photomicrograph (c) of the same patient showing hyaline globules, pseudo-papillae and nuclear grooves confirming the diagnosis of solid pseudo-papillary tumour.

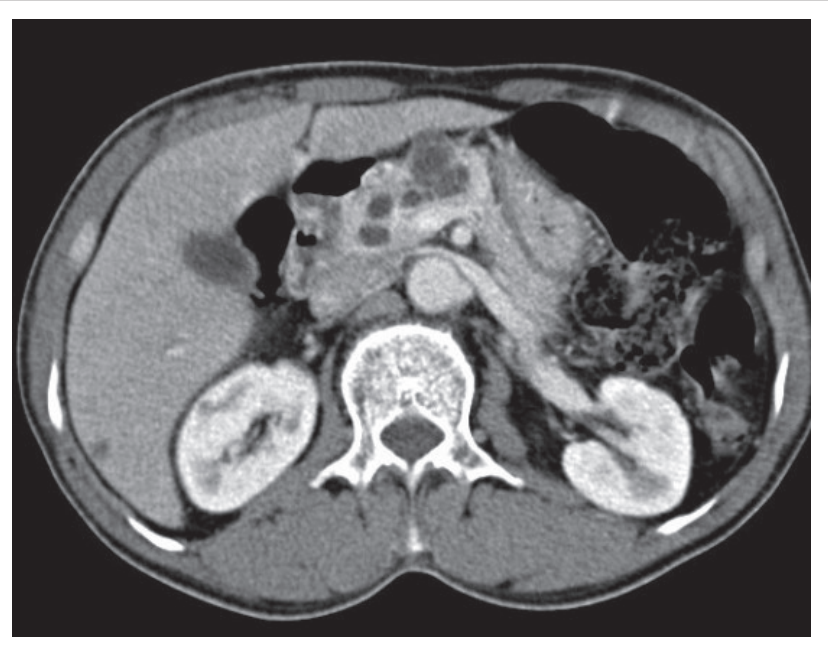

FIGURE 5: Axial contrast-enhanced computed tomography image in a 52-yearold male patient revealing a cystic lesion with septations in the body of pancreas with a dilated main pancreatic duct. A presumptive diagnosis of intra-ductal papillary mucinous neoplasm was made and histopathology examination confirmed the computed tomography diagnosis.

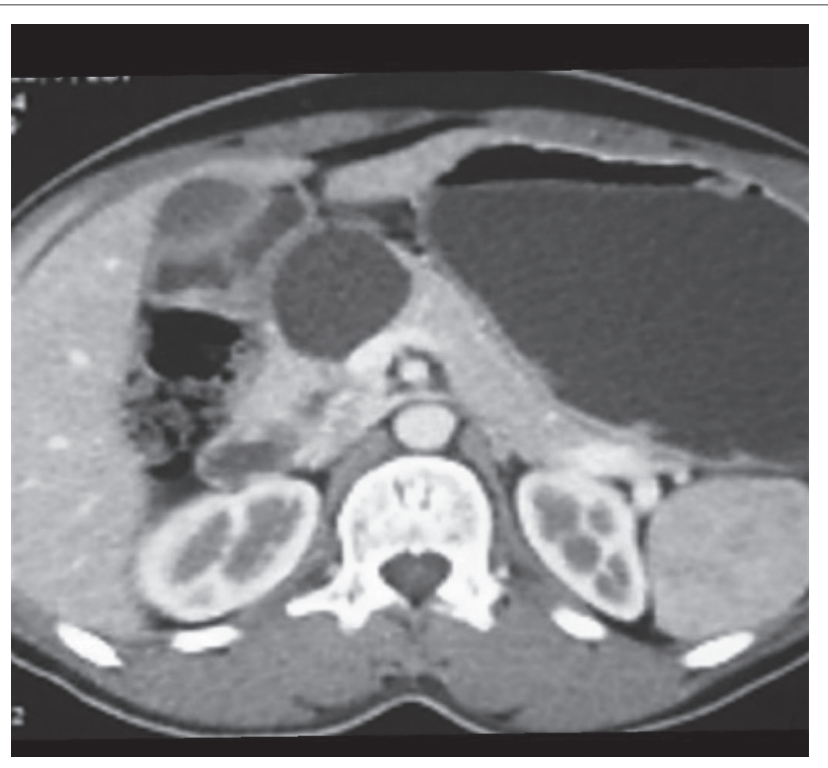

FIGURE 6: Axial contrast-enhanced computed tomography image in a 25-yearold male patient showing a simple pancreatic cyst. No septation or wall enhancement seen. Main pancreatic duct is not dilated. 
Summarising, the overall diagnostic accuracy of CECT in diagnosing pancreatic cystic lesions was $72.5 \%$ in our study. Johnson et al..$^{22}$ were able to definitively diagnose $93 \%$ of serous tumours (14/15 tumours), and Procacci et al. ${ }^{47}$ reported that $\mathrm{CT}$ findings allowed correct characterisation of only $60 \%$ of cystic pancreatic masses.

\section{Conclusion}

The diagnostic accuracy of CT was high in case of SCA, IPMN and pancreatic cysts, and low in case of MCA and SPT. Combination of a multiloculated cystic lesion with locule size of less than $20 \mathrm{~mm}$, septal enhancement with relative lack of wall enhancement, central scar and lobulated outline are highly specific for SCA. Unilocular or macrocystic pattern with locule size of more than $20 \mathrm{~mm}$, female gender and wall enhancement with smooth external contour are pointers towards MCA. Solid cystic pancreatic head lesions in young females may be suggestive of SPT. Dilated MPD in a cystic lesion with internal septations may point towards IPMN. Fluid attenuation lesions with imperceptible non-enhancing wall indicate pancreatic cysts. Lastly, pseudocysts and neuroendocrine tumours with a cystic component are great mimickers of pancreatic cystic lesions, and history of pancreatitis and hormonal profile of patients should always be sought.

\section{Acknowledgements Competing interests}

The authors have declared that no competing interests exist. The views and opinions expressed in this article are those of the authors and do not necessarily reflect the official policy or position of any affiliated agency of the authors.

\section{Author's contributions}

All the authors were involved in data collection, case visits and inference of the data. The writing up of the final article and picture labelling were done by the corresponding author.

\section{References}

1. Kwon RS, Brugge WR. New advances in pancreatic imaging. Curr Opin Gastroenterol. 2005;21(5):561-567.

2. Fernández-del Castillo C, Targarona J, Thayer SP, et al. Incidental pancreatic cysts: Clinicopathologic characteristics and comparison with symptomatic patients. Arch Surg. 2003;138(4):427-434. https://doi.org/10.1001/archsurg.138.4.427

3. Allen PJ, Jaques DP, D'Angelica M, et al. Cystic lesions of the pancreas: Selection criteria for operative and nonoperative management in 209 patients. J Gastrointest Surg. 2003;7(8):970-977.

4. Sheehan MK, Beck K, Pickleman J, et al. Spectrum of cystic neoplasms of the pancreas and their surgical management. Arch Surg. 2003;138(6):657-662. https://doi.org/10.1001/archsurg.138.6.657

5. Delbeke D, Pinson CW. Pancreatic tumors: Role of imaging in diagnosis, staging and treatment. J Hepatobiliary Pancreat Surg. 2004;11(1):4-10. https://doi. org/10.1007/s00534-002-0775-x

6. Kocjan G, Rode J, Lees WR. Percutaneous fine needle aspiration cytology of the pancreas: Advantages and pitfalls. J Clin Pathol. 1989;42(4):341-347.

7. Brugge WR, Lewandrowski $K$, Lee-Lewandrowski $E$, et al. Diagnosis of pancreatic cystic neoplasms: A report of the cooperative pancreatic cyst study. Gastroenterology. 2004;126(5):1330-1336.

8. Centeno BA, Warshaw AL, Mayo-Smith W et al. Cytologic diagnosis of pancreatic cystic lesions. A prospective study of 28 percutaneous aspirates. Acta Cytol. 1997;41(4):972-980. https://doi.org/10.1159/000332775
9. Karmazanovsky G, Fedorov V, Kubyshkin V, et al. Pancreatic head cancer: Accuracy of CT in determination of resectability. Abdom Imaging. 2005;30(4):488-500. https://doi.org/10.1007/s00261-004-0279-z

10. Fusaroli P, Kypraios D, Caletti G, et al. Pancreatico- biliary endoscopic ultrasound: A systematic review of the levels of evidence, performance and outcomes. World J Gastroenterol. 2012;18(32):4243-4256. https://doi.org/10.3748/wjg.v18. i32.4243

11. Dibble EH, Karantanis D, Mercier G, et al. PET/CT of cancer patients: Part 1, pancreatic neoplasms. Am J Roentgenol. 2012;199(5):952-967. https://doi. org/10.2214/AJR.11.8182

12. Tamm EP, Balachandran A, Bhosale $P R$, et al. Imaging of pancreatic adenocarcinoma: Update on staging/resectability. Radiol Clin North Am. 2012;50(3):407-428. https://doi.org/10.1016/j.rcl.2012.03.008

13. Tokar JL, Walia R. Diagnostic evaluation of solid pancreatic masses. Cur Gastroentrol Rep. 2013;15(10):347. https://doi.org/10.1007/s11894-013-0347-z

14. Kim JH, Park SH, Yu ES, et al. Visually isoattenuating pancreatic carcinoma at dynamic-enhanced CT: Frequency, clinical and pathologic characteristics, and diagnosis at imaging examinations. Radiology. 2010;257(1):87-96. https://doi. org/10.1148/radiol.10100015

15. Davenport MS, Viglianti BL, Al Hawary MM, et al. Comparison of acute transient dyspnea after intravenous administration of gadoxetate disodium and gadobenate dimeglumine: Effect on arterial phase image quality. Radiology. 2013;266(2) 452-461. https://doi.org/10.1148/radiol.12120826

16. Pietryga JA, Burke LM, Marin $D$, et al. Respiratory motion artefact affecting hepatic arterial phase imaging with gadoxetate disodium: Examination recovery with a multiple arterial phase acquisition. Radiology. 2014;271(2):426-434. https://doi.org/ 10.1148/radiol.13131988

17. Shaib YH, Davila JA, El-Serag HB. The epidemiology of pancreatic cancer in the United States: Changes below the surface. Aliment Pharmacol Ther. 2006;24(1):87-94. https://doi.org/10.1111/j.1365-2036.2006.02961.x

18. Curry CA, Eng J, Horton KM, et al. CT of primary cystic pancreatic neoplasms: Can $\mathrm{CT}$ be used for patient triage and treatment? Am J Roentgenol. 2000;175(1):99-103. https://doi.org/10.2214/ajr.175.1.1750099

19. Atalay FO, Nesrin U, Berna A, et al. Evaluation of cystic lesions of the pancreas based on clinicopathologic parameters. Acta Medica Mediterranea. 2015;31:383.

20. Parra-Herran CE, Garcia MT, Herrera L, et al. Cystic lesions of the pancreas: Clinical and pathologic review of cases in a five year period. J Pancreas. 2010;11(4):358-364. https://doi.org/10.6092/1590-8577/3621

21. Megibow AJ. Update in imaging of cystic pancreatic masses for gastroenterologists. Clin Gastroenterol Hepatol. 2008;6:1194-1197. https://doi.org/10.1016/j.cgh. 2011.03.005

22. Johnson CD, Stephens DH, Charboneau JW, et al. Cystic pancreatic tumors: CT and sonographic assessment. Am J Roentgenol. 1988;151(6):1133-1138. https://doi. org/10.2214/ajr.151.6.1133

23. Bhatt CJ, Vaishnav K. Multidetector computed tomographic features of uncommon pancreatic masses. NHL J Med Sci. 2014;3(1):316-321. https://doi.org/10.4329/ wjr.v8.i3.316

24. Curry CA, Eng J, Horton KM, et al. CT of primary cystic pancreatic neoplasms: Can CT be used for patient triage and treatment? Am J Roentgenol. 2000; 175(1):99-103. https://doi.org/10.2214/ajr.175.1.1750099

25. Kim SY, Lee JM, Kim SH, et al. Macrocystic neoplasms of the pancreas: CT differentiation of serous oligocystic adenoma from mucinous cystadenoma and https://doi.org/10.2214/AJR.05.0337

26. Torresan F, Casadei R, Solmi L, et al. The role of ultrasound in the differential diagnosis of serous and mucinous cystic tumours of the pancreas. Eur $J$ diagnosis of serous and mucinous cystic
Gastroenterol Hepatol. 1997;9(2):169-172.

27. Balthazar EJ, Chako AC. Computed tomography of pancreatic masses. Am Gastroenterol. 1990;85(4):343-349. https://doi.org/10.1007/s00117-008-1755-1

28. Schulz HU, Kellner U, Kahl S. A giant pancreatic serous microcystic adenoma with 20 years followup. Langenbecks Arch Surg. 2007;392(2):209-213. https://doi. org/10.1007/s00423-006-0146-1

29. Atef E, Nakeeb AE, Hanafy EE, et al. Pancreatic cystic neoplasms: Predictors of malignant behaviour and management. Saudi J Gastroenterol. 2013;19(1):45-53. https://doi.org/10.4103/1319-3767.105927

30. Sahani D, Prasad S, Saini S, et al. Cystic pancreatic neoplasms evaluation by CT and magnetic resonance cholangiopancreatography. Gastrointest Endosc Clin N Am. 2002;12(4):657-672.

31. Warshaw AL, Compton CC, Lewandrowski K, et al. Cystic tumors of the pancreas. New clinical, radiologic, and pathologic observations in 67 patients. Ann Surg. 1990;212(4):432-443.

32. Cohen-Scali F, Vilgrain V, Brancatelli G, et al. Discrimination of unilocular macrocystic serous cystadenoma from pancreatic pseudocyst and mucinous cystadenoma with CT: Initial observations. Radiology. 2003;228(3):727-733. https://doi.org/10.1148/radiol.2283020973

33. Vassos N, Agaimy A, Klein P, et al. Solid-pseudopapillary neoplasm (SPN) of the pancreas: Case series and literature review on an enigmatic entity. Int J Clin Exp Pathol. 2013;6(6):1051-1059.

34. Yoon DY, Hines OJ, Bilchik AJ, et al. Solid and papillary epithelial neoplasms of the pancreas: Aggressive resection for cure. Am Surg. 2001;67(12):1195-1199.

35. Casedei R, Santini D, Calculli L, et al. Pancreatic solid-cystic papillary tumor: Clinical features, imaging findings and operative management. J Pancreas. 2006;7(1):137-144. 
36. Adkisson D, Harris AS, Bridges MD, et al. Solid pseudopapillary tumor of pancreas: Report of five cases. Int J Hepatob Pancreat Dis. 2012;2:9-14. https://doi. org/10.5348/ijhpd-2012-5-CS-3

37. Alves JR, Amico EC. Solid-pseudopapillary neoplasm of the pancreas: Case series and literature review. J Pancreas. 2015;16(3):218-226.

38. Kehagias D, Smyrniotis V, Gouliamos A, et al. Cystic pancreatic neoplasms: Computed tomography and magnetic resonance imaging findings. Int J Pancreatol. 2000;28(3):223-230. https://doi.org/10.1385/IJGC:28:3:223

39. Ye J, Ma M, Cheng D, et al. Solid-pseudopapillary tumor of the pancreas: Clinical features, pathological characteristics, and origin. J Surg Oncol. 2012;106(6):728735. https://doi.org/10.1002/jso.23195

40. Coleman KM, Doherty MC, Bigler SA. Solid-pseudopapillary tumor of the pancreas. Radiographics. 2003;23(6):1644-1648. https://doi.org/10.1148/rg.236035006

41. Choi JY, Kim MJ, Kim JH, et al. Solid pseudopapillary tumor of the pancreas: Typical and atypical manifestations. Am J Roentgenol. 2006;187:178-186. https://doi. org/10.2214/AJR.05.0569
42. Paal E, Thompson LDR, Przygodzki RM, et al. Clinicopathologic and immunohistochemical study of 22 intraductal papillary mucinous neoplasms of the pancreas, with a review of the literature. Mod Path. 1949;12(5):518-528.

43. Adsay NV, Adair CF, Heffess CS, et al. Intraductal oncocytic papillary neoplasms of the pancreas. Am J Surg Pathol. 1996;20(8):980-994.

44. Procacci C, Graziani R, Bicego E, et al. Intraductal mucin-producing tumors of the pancreas: Imaging findings. Radiology. 1996;198(1):249-257. https://doi. org/10.1148/radiology.198.1.8539388

45. Haaga JR, Lanzieri CF, Gilkeson RC. The pancreas. In: Haaga JR, editor. Computed tomography and magnetic resonance imaging of the whole body. 4th ed. Philadelphia, PA: Elsevier, 2003; p. 1395-1485.

46. Shirkhoda A, Mittelstaedt CA. Demonstration of pancreatic cysts in adult polycystic disease by computed tomography and ultrasound. Am J Roentgenol. 1978;131:1074-1106. https://doi.org/10.4103/2303-9027.163001

47. Procacci C, Biasiutti C, Carbognin G, et al. Characterization of cystic tumors of the pancreas: CT accuracy. J Comput Assist Tomogr. 1999;23(6):906-912. 